\title{
Basophil activation testing is useful for the evaluation of life-threatening radiocontrast media anaphylaxis
}

\author{
Jamma Li $^{1}$, Christopher Weir ${ }^{2}$, and Suran Fernando ${ }^{1}$ \\ ${ }^{1}$ Royal North Shore Hospital \\ ${ }^{2}$ The University of Sydney
}

May 26, 2021

Basophil activation testing is useful for the evaluation of life-threatening radiocontrast media anaphylaxis

Jamma Li, MBBS, MPhil (USyd)

Clinical Immunologist and Allergist

Department of Clinical Immunology and Allergy, Royal North Shore Hospital, Reserve Road, St Leonards NSW 2065, Australia

University of Sydney, Sydney, Australia

Jamma.li@health.nsw.gov.au

Christopher Weir, $\mathrm{PhD}$

University of Sydney, Sydney, Australia

Suran Fernando, MBBS, PhD

Department of Clinical Immunology and Allergy, Royal North Shore Hospital, Reserve Road, St Leonards NSW 2065, Australia

University of Sydney, Sydney, Australia

Conflicts of interest: none

Funding source: none

Keywords: allergy, radiocontrast allergy, drug allergy, drug provocation testing, drug challenge

Word Count: 582

Figures: 0

Tables: 2

To the Editor

The mechanism of immediate hypersensitivity reactions (IHR) to radiocontrast media (RCM) is not fully elucidated $(1,2)$. Allergic (IgE-mediated) mechanisms may account for $>50 \%$ of life-threatening IHR, as detected by skin testing (ST) (3). Basophil activation testing (BAT) may be useful for demonstrating allergic sensitisation for severe RCM IHR as there is correlation with ST or drug provocation testing (DPT) without the risk profile (3). ST is relatively standardised compared to BAT. DPT is recommended only for ST-negative RCM (3). 
Our study focusses on the usefulness of BAT for evaluating life-threatening RCM IHR and planning of safe subsequent RCM administration. We sequentially recruited patients at a tertiary institution in Sydney, Australia between July 1, 2019 and June 30, 2020. All patients experienced symptoms consistent with Grade 3 or 4 anaphylaxis within 1 hour of RCM administration (4). Clinical characteristics and mast cell tryptase levels were collected. Testing was performed to RCMs available in our institution; iodixanol (Visipaque, GE Healthcare Australia Pty Ltd, Sydney, Australia), iohexol (Omnipaque, GE Healthcare Australia Pty Ltd), iopromide (Ultravist, Bayer Australia Ltd, Sydney, Australia), meglumine iotroxate (Biliscopin, Bayer Australia Ltd), and sodium diatrizoate and meglumine amidotrizoate (Urografin, Bayer Australia Ltd). ST was performed to EAACI guidelines (1). BAT was performed in line with our protocol (5) at 1:10000-1:10 dilutions. Results were expressed as percentage upregulation above the negative control, and stimulation index (SI) for CD63 and CD203c. If ST to Urografin was negative, the patient proceeded to single-dose oral sodium diatrizoate 100mg and meglumine amidotrizoate 660mg DPT (Gastrografin 100mL, Bayer Australia Ltd) with no premedication. Patients then proceeded to routine RCM administration for contrast radiography, or $0.5 \mathrm{ml} / \mathrm{kg}$ single-dose intravenous RCM challenge with alternative suitable STnegative $\mathrm{RCM} / \mathrm{s}$ with no premedication.

Patient characteristics are shown in Table 1. Ten study patients were enrolled, the culprit RCMs were iohexol $(n=5)$, iopromide $(n=2)$, and amidotrizoate $(n=1)$. Two patients did not have RCM anaphylaxis and reserved as controls (RCM extravasation, and vasovagal syncope). Findings are shown in Table 2. Six (75\%) had significant change in mast cell tryptase. Five (62.5\%) had positive ST to the culprit RCM, all had significant tryptase change. Using standard cut-offs for BAT of 5\% and 10\% upregulation for CD63 and CD203c respectively, and a SI $>2.0(5,6)$, there were 4 false positives to amidotrizoate, and 1 to iohexol. Adjusting cut-offs to $10 \%$ and $15 \%$ reduced false positives to 2 for amidotrizoate, and 0 for iohexol. BAT was positive to the culprit RCM in 5 patients (62.5\%), including 2 ST-negative patients. Six of $8(75 \%)$ were only positive to the culprit drug on ST and BAT. Both control patients had negative ST and BAT. There was significant lack of agreement between ST and BAT $(\mathrm{P}<0.05)$ (McNemar's test).

Our case series centres on patients with life-threatening RCM IHR. Seven of $8(87.5 \%)$ had a positive ST or BAT suggesting that most Grade 3 or 4 anaphylaxis has an allergic mechanism. Also, the patient with negative ST and BAT was evaluated remotely (1706 days from reaction). In this study, BAT was a useful adjunct to ST as it identified an extra 2 patients with allergic IHR. BAT was not useful for amidotrizoate with a significant false positive rate $(29 \%)$.

Therefore, most life-threatening RCM IHRs may have an allergic mechanism. This is significant, as in allergic IHR there may be less cross-reactivity with other RCM. BAT may be a useful adjunct to ST to demonstrate this allergic mechanism. Further study can optimise performance characteristics of BAT, especially for amidotrizoate.

Contribution: JL, CW performed the experiments; J.L, CW, SF analysed the results and constructed the tables; JL, CW and SF developed and designed the research; JL wrote the paper.

1. Torres M, Trautmann A, Bohm I, et al. Practice parameters for diagnosing and managing iodinated contrast media hypersensitivity. Allergy 2021;76:1325-39.

2. Brockow K, Romano A, Aberer W, et al. Skin testing in patients with hypersensitivity reactions to iodinated contrast media - a European multicenter study. Allergy. 2009;64:234-41.

3. Clement O, Dewachter P, Mouton-Faivre C, et al. Immediate Hypersensitivity to Contrast Agents: The French 5-year CIRTACI Study. EClinicalMedicine 2018;1:51-61.

4. Ring J, Messmer K. Incidence and severity of anaphylactoid reactions to colloid volume substitutes. Lancet 1977;1:466-9.

5. Li J, Best O, Rose M, Green S, Fulton R, Fernando S. Integrating basophil activation tests into evaluation of perioperative anaphylaxis to neuromuscular blocking agents. Br J Anaesth. 2019;123:e135-43. 
6. Pinnobphun P, Buranapraditkun S, Kampitak T, Hirankarn N, Klaewsongkram J. The diagnostic value of basophil activation test in patients with an immediate hypersensitivity reaction to radiocontrast media. Ann Allergy Asthma Immunol. 2011;106:387-93.

Table 1. Patient characteristics

\begin{tabular}{|c|c|c|c|c|c|c|c|c|c|c|c|}
\hline Patient & $\begin{array}{l}\text { Gender } \\
(\mathrm{M} / \mathrm{F})\end{array}$ & $\begin{array}{l}\text { Age, } \\
\text { years }\end{array}$ & $\begin{array}{l}\text { Time } \\
\text { from } \\
\text { reac- } \\
\text { tion, } \\
\text { days }\end{array}$ & $\begin{array}{l}\text { Contrast } \\
\text { agent } \\
\text { ad- } \\
\text { minis- } \\
\text { tered }\end{array}$ & $\begin{array}{l}\text { Sympt } \\
\text { of } \\
\text { IHR }\end{array}$ & $\begin{array}{l}\text { Grade } \\
\text { of } \\
\text { mata- } \\
\text { phy- } \\
\text { laxis }\end{array}$ & $\begin{array}{l}\text { Asthma } \\
\text { (Yes/No }\end{array}$ & $\begin{array}{l}\text { Atopy } \\
\text { (Yes/No }\end{array}$ & $\begin{array}{l}\text { Severe } \\
\text { car- } \\
\text { dio- } \\
\text { vascu- } \\
\text { lar } \\
\text { dis- } \\
\text { ease } \\
\text { (Yes } / \mathrm{No}\end{array}$ & $\begin{array}{l}\text { Previous } \\
\text { RCM } \\
\text { expo- } \\
\text { sure } \\
(\text { Yes } / \mathrm{No})\end{array}$ & $\begin{array}{l}\text { s Previous } \\
\text { RCM } \\
\text { reac- } \\
\text { tion } \\
(\text { Yes } / \mathrm{No})(\end{array}$ \\
\hline 1 & $\mathrm{~F}$ & 37 & 105 & Iohexol & $\begin{array}{l}\mathrm{CV}, \mathrm{G}, \\
\mathrm{S}\end{array}$ & 3 & No & No & No & Yes & $\begin{array}{l}\text { Yes } \\
\text { (Flushing) }\end{array}$ \\
\hline 2 & $\mathrm{~F}$ & 61 & 242 & Iohexol & $\begin{array}{l}\mathrm{CV}, \\
\mathrm{R}, \mathrm{S}\end{array}$ & 3 & Yes & Yes & No & No & No \\
\hline 3 & $\mathrm{~F}$ & 69 & 50 & Iohexol & $\mathrm{R}, \mathrm{S}$ & 3 & No & Yes & Yes & No & No \\
\hline 4 & M & 44 & 455 & Iohexol & $\begin{array}{l}\mathrm{CV} \\
\mathrm{R}\end{array}$ & 3 & No & No & Yes & No & No \\
\hline 5 & $\mathrm{~F}$ & 75 & 371 & Iohexol & $\mathrm{CV}, \mathrm{R}$ & 3 & No & Yes & No & Yes & $\begin{array}{l}\text { Yes } \\
\text { (Vomiting) }\end{array}$ \\
\hline 6 & $\mathrm{M}$ & 83 & 1706 & \multicolumn{2}{|c|}{$\begin{array}{r}\text { IopromideCV, } \\
\mathrm{R}\end{array}$} & 4 & No & No & Yes & Yes & No \\
\hline 7 & $\mathrm{~F}$ & 74 & 404 & \multicolumn{2}{|c|}{$\begin{array}{c}\text { IopromideCV, } \\
\mathrm{S}\end{array}$} & 3 & No & No & No & No & No \\
\hline 8 & $\mathrm{M}$ & 40 & 376 & \multicolumn{2}{|c|}{$\begin{array}{c}\text { AmidotrizGate } \\
\text { S }\end{array}$} & 3 & No & No & No & No & No \\
\hline
\end{tabular}

* CV: cardiovascular (hypotension, tachycardia); R: respiratory (desaturation, symptoms of airway obstruction including stridor, throat tightness, dysphonia, cough, wheeze), S: skin (urticaria, angioedema), G: gastrointestinal (nausea, vomiting, abdominal discomfort, diarrhoea)

IHR, immediate hypersensitivity reaction

$\mathrm{RCM}$, radiocontrast media 


\begin{tabular}{|c|c|c|c|c|c|c|c|c|c|c|c|c|c|}
\hline & & $\mathrm{ST}^{*}$ & $\mathrm{ST}^{*}$ & $\mathrm{ST}^{*}$ & $\mathrm{ST}^{*}$ & $\mathrm{ST}^{*}$ & BAT* $^{*}$ & BAT* & BAT* & BAT* & BAT* & $\begin{array}{l}\text { DPT } \\
\text { or } \\
\text { ther- } \\
\text { a- } \\
\text { peu- } \\
\text { tic } \\
\text { ad- } \\
\text { min- } \\
\text { is- } \\
\text { tra- } \\
\text { tion }\end{array}$ & $\begin{array}{l}\mathrm{DP}^{\prime} \\
\text { or } \\
\text { ther } \\
\text { a- } \\
\text { peu } \\
\text { tic } \\
\text { ad- } \\
\text { min } \\
\text { is- } \\
\text { tra- } \\
\text { tion }\end{array}$ \\
\hline & $\begin{array}{l}\text { Tryp } \\
\text { sig- } \\
\text { nifi- } \\
\text { cant } \\
\text { rise } \\
\text { and } \\
\text { fall } \\
\text { (Yes }\end{array}$ & To) & Iodix & ddbpro & $\begin{array}{l}\text { iddeglur } \\
\text { iotrox- } \\
\text { ate }\end{array}$ & $\begin{array}{l}\text { nAfeglu } \\
\text { ami- } \\
\text { dotri- } \\
\text { zoate }\end{array}$ & nInhexol & Iodixa & dbpron & $\begin{array}{l}\text { ideeglun } \\
\text { iotrox- } \\
\text { ate }\end{array}$ & $\begin{array}{l}\text { aMeglu } \\
\text { ami- } \\
\text { dotri- } \\
\text { zoate }\end{array}$ & AInhexo & Iodi \\
\hline 1 & Yes & + & - & - & - & - & - & - & - & - & + & Culprit & \\
\hline 2 & Yes & + & - & - & - & - & - & - & - & - & - & Culprit & \\
\hline 3 & No & - & - & - & - & - & + & + & + & + & - & Culprit & $\mathrm{n} / \mathrm{d}$ \\
\hline 4 & Yes & + & + & - & - & - & + & + & + & + & + & Culprit & $\mathrm{n} / \mathrm{p}$ \\
\hline 5 & Yes & + & - & - & - & - & + & - & - & + & + & Culprit & $\mathrm{n} /$ \\
\hline 6 & Yes & - & - & - & - & - & - & - & - & - & - & Pass & \\
\hline 7 & $\mathrm{n} / \mathrm{p}$ & - & - & - & - & - & - & - & - & + & + & Pass & $\mathrm{n} /$ \\
\hline 8 & Yes & - & - & - & - & + & - & - & - & - & + & Pass & $\mathrm{n} /$ \\
\hline
\end{tabular}

Table 2. Results of ST, BAT and DPT to RCM

* ST and BAT was performed to iohexol, iodixanol, iopromide, meglumine iotroxate, and sodium diatrizoate and meglumine amidotrizoate for all patients.

ST, skin testing

BAT, basophil activation test

RCM, radiocontrast media

$\mathrm{n} / \mathrm{p}$, not performed

$\mathrm{n} / \mathrm{d}$, not done as patient declined 